\title{
AVALIAÇÃO CRITICA DA PSIQUITRIA COMUNITARIA
}

CLOVIS MARTINS *

PAULO CESAR SANDLER **

As avaliações que se seguem implicam em juízos de valor a respeito do "movimento" da psiquiatria comunitária tentando cobrir o plano das abstrações teóricas e de suas implicações práticas. Como postura inicial devemos reconhecer certas dificuldades para uma análise rigorosamente objetiva do tema: 1) a psiquiatria comunitária se constitui em campo de investigação recente de limites imprecisos e mal definidos $1,5,7,14,50 ; 2)$ faltam-nos conceitos precisos 45 e os dados reunidos em trabalhos de investigação a respeito são muitas vezes contraditórios ${ }^{57}$ quando não inconclusivos ${ }^{2,54}$. O presente estudo, de pretensões críticas, foi realizado sobre conceitos operacionais, numa tentativa de abranger amplamente aspectes que aparecem na literatura a respeito. Usaremos a expresão "psiquiatria comunitária" como substitutiva de "saúde mental comunitária", conforme proposto em trabalhos anteriores ${ }^{49}$ já que ainda não existe acordo sobre o campo semântico destes têrmos 4 .

O estudo será desenvolvido em níveis de abstração, enfocando três ordens de grandeza: inicialmente tentaremos alcançar o macro-nível (o "sociológico"), passando pelo nível das interações (que pode ser chamado de "análise microsociológico"), chegando, finalmente, no micro-nivel (o "individual"). Em cada um deles focalisaremos pontos controversos na teoria e na prática da psiquiatria comunitária, que podem ser imputados como responsáveis por inconsistências em suas posições e/ou fracassos em suas execuções.

\section{CONCHITUACOES TEORICAS F PRATICAS}

A psiquiatria comunitária pode ter seu campo delimitado como um meio caminho entre a teoria e a prática ${ }^{43.49}$. Certas idéias que a nutrem são provenientes de um campo teórico puro: a psiquiatria social 43,48,58. A teoria em psiquiatria comunitária, embora admita certas leis generalizadoras é intimamente ligada e dependente da prática: não existiria uma psiquiatria comunitária, mas tantas quantas sejam os locais a que elas se destinam. As necessidades (psiquiátrica) destes locais têm sido dadas a conhecer pelo método epidemiológico $6.14 .37,50,51$.

Trabalho apresentado no temário oficial do XII Congresso Brasileiro de Neuroligia, Psiquiatria, Higiene Mental, Brasilia, 1975: *Docente Livre de Psiquiatria da Faculdade de Medicina da Universidade de São Paulo; ** Psiquiatra supervisor de treinamento na Faculdade de Saúde Pública da Universidade de săo Paulo. 
Pode-se afirmar que o fulcro da teoria que nutre a psiquiatria comunitária é um interesse pelo meio ambiente ${ }^{5,45,49}$. Este apoio teórico básico, propositalmente formulado de maneira vaga, diz respeito a uma nova ideologia na compreensão e manejo do doente mental: não se aceita mais a abstração cartesiana do "doente mental" como entidade individualizável ${ }^{13}$, mas substentase que sua compreensão científica só é possível quando esta abstração é enriquecida com um ponto de referência interacional 48 .

O modelo médico clássico, de tradição positivista ${ }^{1,17}$, o qual deu margem ao conceito de doente mental como sede de uma determinada doeça, é suplementado. Este ponto de referência interacional reflete a forte influência do modelo psicodinâmico, que se faz sentir também nas hipóteses causais e etiopatogênicas dos problemas mentais. Como já notado, as relações simples de causa e efeito não são admitidas, e a dinâmica causal, multifatorial, não é mais exaustivamente procurada apenas no mundo interno dos indivíduos, mas esta procura estender-se ao meio social, ao ambiente como um todo 2,49,58. Os

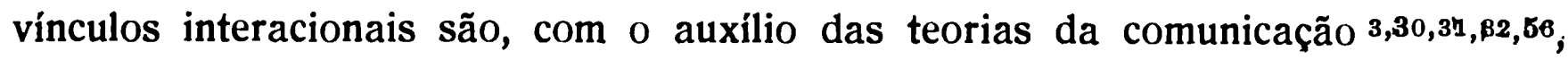
focalizados e neles também se isolam aspectos patológicos. A própria organização social começa a ser colocada como sede da doença $2,18,53$ : o meio social é encarado como patológico, ou a dinâmica social, como doente 2.23 .

Assim podemos dispor atualmente de uma série de conjecturas e hipóteses, algumas poucas verificações empíricas que nem mesmo chegam a formar um verdadeiro corpo de doutrina sobre fatores sociais e doença mental ${ }^{49}$. O fulcro da teoria é uma pergunta: tem a cultura, o meio social, alguma relação com a doença mental 20? há algum fator social observável associado aos problemas dos quais cuida a psiquiatria? Através de métodos emprestados de outras ciências pode-se afirmar que há, mas o aproveitamento prático deste pequeno conhecimento é prejudicado pela falta de um modelo teórico próprio que explique os mecanismos das relações entre fatores ambientais e doença mental $2.18,23,24,35,4: 2,48,49,58$.

O movimento da psiquiatria comunitária tem sido qualificado de "revolucionário" dentro da história da psiquiatria e mesmo fora dela $5,7,14,23,45,40$. $O$ interesse pelo meio ambiente caracterizaria uma revolução no campo das idéias e sua consequência mais imediata, que tem soado como revolucionária, é uma alternativa institucional às técnicas psiquiátricas tradicionais. Decorrente da posição teórica, a atenção não é voltada para o indivíduo em si, mas para toda uma população $5,6,25,29,44,50$ a qual, com nome de comunidade, tem sido entendida em termos meramente geográficos ${ }^{5,49}$. A responsabilidade não é do médico sobre o paciente, mas sim da organização social (que nomeia e investe de poder legal seus representantes) sobre uma parcela da população identificada como de comportamento desviado.

Como fenômeno de nosso tempo, as técnicas da medicina preventiva são trazidas à psiquiatria, já que o tratamento curativo tradicional, ou seja, o confinamento dos doentes em hospícios provou ser inútil em termos terapêuticos e custoso em termos sócio-econômicos ${ }^{34}$. As técnicas preventivas se constituem com o psiquiatra se envolvendo profundamente na dinâmica social, cabendo-lhe 
um papel de instrutor para certos leigos considerados pessoas-chave na comunidade, os "agentes naturais da saúde mental"', com vistas à eliminação de condições ambientais nocivas. $\mathrm{O}$ antigo envolvimento "médico-paciente" passa a ser o "moderno" "instituição-população". O psiquiatra teria ainda seu papel de organizador desta instituição, dela fazendo parte as tradicionais profissões paramédicas, com papel ativo junto ao doente, além de cientistas sociais, administradores de empresas e economistas ${ }^{44}$.

A longiqua instituição asilar, alienada e alienante, onde os rotulados de doentes eram custodiados, deixa de ser a viga mestra: instituem-se postos avançados no seio da comunidade, harmonizados com sua dinâmica e dela fazendo parte: os chamados centros de saúde mental comunitária, advogando-se sua integração com o sistema de saúde global. O confinamento cede lugar ao tratamento ambulatorial, havendo numerosas referências à importância dos psicotrópicos e da grupoterapia 11 na "terceira revolução psiquiátrica". Os centros devem estar perto do lar do paciente, para evitar "uma retirada da pessoa de seu convívio e do contato normais com sua família, trabalho e escola", impedindo sua alienação.

Técnicas psicoterápicas específicas são desenvolvidas, mostrando-nos a literatura como se pode fazer psicoterapia em pessoas de baixa renda econômica, as mais expostas ao risco e principais beneficiárias da psiquiatria comunitária, tornando-se uma constante a exaltação do papel "progressista"12 das psicoterapias breves.

A psicoterapia comunitária acabou por encampar instituições já existentes, que foram inclusive suas origens, de formas, complexidade e objetivos variáveis, tais como o day-hospital, as oficinas abrigadas, as halfway houses, as walk-in clinics, os centros comunitários administrativos, os hospitais de custódia, e o hospital comunitário modelo: a comunidade terapêutica $5,6,7,8,12,14,19,25,26,38,49,50,52,59$.

\section{AVALIAÇAO CRITICA}

Nivel "sociológico" de observação (o "macro-nivel"49) - As conotações revolucionárias do movimento podem estar sendo errôneas. Elas levam a radicalizações que prejudicam a compreensão do fenômeno chamado pela psiquiatria de doença mental. Ao invés das novas idéias serem encaradas como suplementares, elas têm adquirido um valor exclusivista: é a sociedade que causa as doenças; as teorias intrapsíquicas e do meio interno são "erradas" e "ultrapassadas". E proposta a substituição da "psicodinâmica" por uma "sociodinâmica". Intromissões políticas são vistas: a procura de fatores sociais é "progressista"12 ou "subversiva"16, e a psicodinâmica e a psicopatologia clínica, ciências "burguesas" e "reacionárias"*. Acreditamos que ambas as aproximaçõões, se integradas, oferecem uma abertura a mais para a compreensão do enigma da

- Pode-se consultar, por exemplo, "Is Community Psychiatry a Communist Plot", ed. pelos lab. Smith Klne \& French, EUA, 1967; "La Medicina Soviética en Lucha contra las Enfermedades Mentales", de L. Rojlin, sem data, editado pelo governo russo. 
doença mental, do que se encaradas como exclusivas: as teorias sociogenéticas da doença não são intrinsecamente opostas aos conhecimentos já disponniveis 10,16,41,46,47,49. A demais, todo o "movimento" da psiquiatria comunitária só aparece depois de legalizado e institucionalizado, o que nos parece pouco revolucionário 49 .

$\mathrm{Na}$ verdade, estas medidas são revolucionárias ou mantenedoras de um "status quo"? Muitos ${ }^{5}$ notam que a psiquiatria comunitária implica na extensão dos cuidados psiquiátricos à parcela da população que até então não tinha acesso a eles.

Até que ponto tal extensão é genuinamente psiquiätrica? Ao se responsabilizar de maneira direta pelos menos favorecidos economicamente, a psiquiatria comunitária tenta implantar os conhecimentos psiquiátricos oriundos das classes dominantes sobre a parcela da população que não tinha contato com estes conhecimentos 5,16. Até que ponto esta visão aparentemente humanitária, médica, não está a encobrir uma prática de controle social 15? Não devemos nos esquecer que é a sociedade quem indica ao médico os doentes que ele deve tratar 2,15 , e na maioria das vezes, especialmente em organizações sociais (que podemos chamar de tecnocráticas) como a nossa, onde há um estímulo de aspectos competitivos e de alta produção, as pessoas aparecem como doentes e passam a não mais ser suportadas em seu meio de origem na medida em que se tornam um ônus econômico.

E quando o psiquiatra sai a campo, e se defronta com os graves problemas sociais? Parece ter nascido aí a sociatria ${ }^{36}$, advogando experimentações maciças, baseadas na experiência psiquiátrica, afetando a engrenagem social. Os limites das idéias políticas de cada profissional e de sua prática enquanto psiquiatra passam a ser tênues e imprecisos. A psiquiatria comunitária seria um meio de reforma social em situações e comunidades malsãs $2,16,25,36$. Em suma: o psiquiatra acumularia funções revolucionárias, em termos sociais. Esta perspectiva de atuação nos parece viável para qualquer cidadão, mas existem questionamentos éticos e morais quando a pessoa age como profissional médico $16,36,46,55$.

$\mathrm{Na}$ verdade, podem existir explicações a nivel profundo para tais opções, como veremos a seguir.

Nivel intermediário, análise das interações (ou "micro-sociológico") - Podemos isolar uma série de sub-sistemas, compostos, a um nível, das entidades institucionalizadas e, em outro, dos profissionais que nelas atuam e a compoem. Ao interagir, estes sistemas esbarram, como lei geral, com uma série de contradições, que podem ser responsabilizadas por obstáculos na execução das idéias da psiquiatria comunitária.

Os cientistas sociais, que por motivos óbvios se interessaram pelo meio ambiente muito antes que os psiquiatras $2,0,18,28,20,40,53,54,58$ dizem que as relações entre doença mental e estrutura social são assunto seu, encarando o psiquiatra como um intrometido, e pior ainda, desinformado para tal pretensão. Sugerem uma sociologia psiquiátrica, ao invés de uma psiquiatria social 2,54. 
E os fatos parecem lhes dar razão, pois até o momento não dispomos de qualquer modelo teórico puro da psiquiatria social; como já notado, existem conjecturas e hipóteses, baseadas, na maior parte das vezes, em conceitos durkheinianos 9,23: os conhecimentos empíricos são esparsos, não unificados, obtidos por metodologia sempre emprestada ${ }^{48}$. Os sociólogos reclamam da "ditadura" imposta pelos psiquiatras nas equipes multidisciplinares sobre 0 rumo e o teor das pesquisas ${ }^{2,30}$. Este tipo de crítica parte dos cientistas que têm sido, a bem verdade, os responsáveis pelo pouco conhecimento disponivel53,18 e também se verifica na prática: as equipes dos centros de saúde mental têm funcionado sob a tutela de pensamentos exclusivamente psiquiátricos. Pensam os cientistas sociais que as grandes potencialidades e esperanças da saúde mental comunitária estão sendo postas a perder por uma visão estreita que nunca passa dos limites psiquiátricos clássicos. Afirmam que a integração harmônica no seio da comunidade tem sido difícil pelo fato dos centros não passarem de um "quisto psiquiátrico", uma extensão da ideologia psiquiátrica ${ }^{39}$ com os velhos métodos de consulta clínica revestidos de novas fachadas, mas sem mudanças de essência, e encerrando-se ai.

Os serviços que integram os sistemas de psiquiatria comunitária, centıalizados administrativamente, constituem uma rêde, complicada e extensa, socialmente orientada nas suas linhas de trabalho junto à população. Os profissionais das diferentes disciplinas que compoem a equipe psiquiátrica vivem aí numa constante e dinâmica interpenetração nas respectivas áreas de atividade. Por isto, cada um deles luta por manter sua autoridade para preservar a identidade profissional e do respectivo campo de atuação ${ }^{27}$. Já na Comunidade Terapêutica, a identidade é absorvida pelas forças emergentes de atuação grupal, inexistindo campo de ação técnica para cada profissional (seria o verdadeiro "role-blurring"). Estes aspectos tornam a inserção da Comunidade Terapêutica nos sistemas de atendimento integrados nos princípios da Psiquiatria Comunitária, difícil de ser obtida ${ }^{27}$.

A Comunidade Terapêutica tem de ter grande autonomia para dominar por completo o programa que põe em execução. O Centro Comunitário de Saúde Mental não permite tal autonomia a nenhum dos serviços que com ele se relacionam. Um exemplo das colisões e das dificuldades resultantes desta disparidade começa com a forma de admissão dos pacientes para tratamento hospitalar. A unidade de internação psiquiátrica integrada em um sistema de assistência comunitária dificilmente poderá ser seletiva. Neste tipo de trabalho assistencial não é mais possível supormos que o paciente descartado pelo profissional que o atende (nem sempre um psiquiatra) vá encontrar amparo em outra unidade psiquiátrica ou em um hospital qualquer, uma vez que a rede incorpora todos os recursos da área e tem que dar cobertura total à população da região sob sua responsabilidade. A heterogeneidade das internações daí resultantes não é facilmente acomodada em uma Comunidade Terapêutica de organização ortodoxa: dentre os pacientes admitidos, muitos não terão condições de assumir responsabilidades no grupo e irão requerer controle medicamentoso e proteção maiores que os outros conforme suas necessidades. A própria rotatividade dos pacientes adquire um ritmo que torna muito mais 
instável o grupo em causa: a tomada de responsabilidade e a participação nas decisões grupais pelos pacientes que ali permanecem por curto período de tempo tornam-se superficiais, quando não impraticáveis. Nos grupos em que esta rotatividade está muito acelerada e cujas admissões escapem do poder de decisão da Comunidade Terapêutica, invariavelmente os técnicos mais diferenciados da equipe acabam por tomar as rédeas, contrariando outro princípio essencial da Comunidade Terapêutica, que é desta conduzir-se sempre pelas decisões consensuais tomadas pelo grupo ${ }^{2 \tau}$.

Nivel individual ou intrapsiquico ("micro-nivel") de observação - Certas idéias da psiquiatria comunitária sobre os papéis reservados ao psiquiatra podem revelar aspectos profundos da personalidade e de quem os adota aprioristicamente e "sem resistência"55. $O$ isolamento em certas posições e opções com um afastamento das funções da prática do psiquiatra, ou seja, suas funções médicas, merecem considerações à parte.

As idéias de uma sociatria, de um papel pedagógico, a absorção dos psiquiatras em funções burocráticas de organização, a chefia dos locais onde eles trabalham, distanciam estes técnicos altamente especializados de seus doentes.

Terminando-se com a relação "terapêuta-paciente", entendendo-o como "instituição-população", ocorre que outros profissionais são tolerados para assumir as responsabilidades tradicionalmente médicas que implicam no contato direto com o paciente: o psicólogo, o assistente social, o laborterapeuta, a nova profissão dos "trabalhadores de saúde mental" levam a cabo os trabalhos de redigir anamneses (que acabam se tornando formulários a serem preenchidos) e cuidar das psicoterapias. Se não nos deixarmos levar por racionalizações fáceis, como a "falta de pessoal", ou que "é um conceito retrógrado" e "chauvinista" que somente médicos podem fazer estes trabalhos, é fácil ver que a consequência é que o médico acaba se afastando do paciente. $O$ mais grave destes aspectos é que nada se faz para legalizar o trabalho destes profissionais em suas "novas" funções, ou, o que é pior ainda, se lhes dê a devida formação para tais tarefas 20 .

E aqui que aparecem cerradas barragens de juízos de valor: uma pessoa que dedique sua vida profissional a algumas dúzias de pacientes é "elitizante" e alienada. Em vez disto, o "progressista" é prevenir que adoeçam milhões de pessoas. Esta alternativa é aparentemente humanitária, sem dúvida fascinante e grandiosa. Mas porque é encarada como a alternativa e não recurso suplementar? Até que ponto não está a situação geográfica dos asilos, com os locais da equipe psiquiátrica bem distantes e guardados dos pavilhões, colônias e enfermarias, com pesados portões para garantir a separação?

Sem dúvida, o contato com a doença, com o sofrimento e com a miséria humana são experiências dolorosas. E talvez o contato com o paciente psiquiátrico seja o mais doloroso, na medida em que ele faça reviver no médico as suas próprias experiências e dificuldades não resolvidas ${ }^{20}$. Como na imagem feita por Kubie, tocar em um paciente psiquiátrico é como tocar em um fogão quente: a reação imediata é tirar a mão, instantaneamente. 
Merece ainda cuidado a idéia de "instrutor" e de chefe da equipe multidisciplinar que se reserva ao psiquiatra. Mesmo que o psiquiatra-instrutor vá lidar com os "agentes naturais de saúde mental", autóctones e espontâneos da comunidade, até que ponto ele pode ensiná-los e instrui-los? Até que ponto ele é que deveria ser ensinado ${ }^{44}$ ? $O$ conhecimento cientifico (estamos encarando a psiquiatria como ciência) é uma parcela pequena do conhecimento dos fenômenos: aquilo que os psiquiatras chamam de doença mental é muitas vezes compreendido de maneira totalmente diversa (desconhecida para nós, psiquiatras) pela população ${ }^{36}$. Encara-se hoje como uma das grandes lições e inovações dos centros comunitários remanescentes, um envolvimento e integração orgânica com a população de tal ordem que, para ilustrar com um exemplo extremo, a sua própria chefia passou para a mão de leigos. Por outro lado, alguns foram depredados pela população, e um grande e melancólico número dos que foram entusiasticamente abertos, estão fechados atualmente. Esta premissa pedagógica também é grandiosa, revelando, talvez, traços de onipotência de quem as adota (incluímos aqui o psiquiatra-reformador social que cuida de milhões, o psiquiatra exclusivamente chefe-de-equipe). Julgamos oportuno notar que, em nosso meio, parece haver uma certa preocupação das vantagens dos manejos e medidas em relação aos comportamentos desviados que provêm da comunidade, como as utilizações de práticas para-religiosas, muitas vezes integradas como recursos no planejamento de serviços. Mesmo assim, tal visão pode ser posta a perder se o psiquiatra permanecer com um papel e status "superior"; a própria palavra "utilização" nos parece suspeita, como se estas práticas já não estivessem sendo utilizadas muito antes dos médicos por elas se interessarem, ou não fossem continuar sendo após o seu "aproveitamento".

\section{CONSIDERACOES FINAIS}

Marcar as limitações e contradições internas da psiquiatria comunitária é uma contribuição para seu aperfeiçoamento. São inegáveis suas enormes potencialidades para um tratamento e compreensão eficazes e humanos das pessoas apontadas como doentes mentais, independentemente de suas possibilidades econômicas. Não obstante, qualquer adesão rígida, dogmática, moralizadora e exclusivista às novas idéias é perigosa. Os resultados desta adesão são previsiveis, pois não terão qualquer contato com a essência destas idéias, mas sim com a atitude em si mesma; estes resultados serão os mesmos que se verificam quando existe este tipo de adesão a qualquer idéia, velha ou nova, sempre en detrimento daquele que será (ou continuará sendo) o grande esquecido: o doente mental.

RESUMO

O presente estudo desenvolve alguns juízos de valor em relação ao movimento da psiquiatria comunitária, em uma tentativa de contribuir para o aperfeiçoamento de sua prática. Através de conceituações teóricas da psiquiatria 
social e da psiquiatria comunitária, os autores sintetizam os pontos básicos que formam o corpo de doutrina e sobre os quais se fazem as avaliações críticas, em três níveis de observação: o nível sociológico, no qual são examinadas as conotações revolucionárias da psiquiatria comunitária e sua inserção como instrumento de controle social em dada organização e estrutura social: o nível intermediário, a análise das interações, aonde são examinadas as reais contribuições, em termos de avancos nos cuidados aos doentes, de todo o movimento, e também aspectos ligados às comunidades terapêuticas; e, finalmente, o nível intrapsíquico, no qual são examinadas as possíveis motivações profundas dos profissionais que aderem ao movimento. São feitas ainda considerações sobre problemas ligados à formação de pessoal "leigo" que precisa assumir atribuições tradicionalmente denominadas médicas.

\section{SUMMARY}

\section{Critical evaluations on community psychiatry}

The present study is a critical evaluation on community psychiatry concepts and practice, in a effort to improve it. The authors point basic aspects on social psychiatry through theoretical issues and make the evaluations by the use of three levels: 1st) the sociological level, where they examine revolutionary adjectives and connotations commonly given to community psychiatry and its role as a social control weapon; 2nd) the intermediate level (interaction analysis), where they examine the real contributions, in terms of advancements in pacients care and also some therapeutic community-linked aspects; and the 3rd), the intrapsychic level, where they examine inner needs and motivations that may be found in a professional commitment to this field. There are some comments on laymen's work when they are commited with a tradicionaly medical area.

\section{REFERFNCIAS}

1. AdORNO, T. W. - La Disputa del Positivismo en la Sociologia Alemana. Versão castelhana. Ed. Grijalho, Barcelona, 1973.

2. BAstide, R. - Sociologia das Doenças Mentais. Versăo brasileira. Comp. Ed. Nacional, Sשo Paulo, 1967.

3. BATESON, G.; JACKSON, D.; HALEY, J. \& WEAKLAND, J. - Toward a theory of schizophrenia. Behav. Sci. 1:251, 1956.

4. BELL, N. W. \& SPIEGEL, J. P. - Social psychiatry: vagaries of a term. Arch. Gen. Psychiat. 13:337, 1966.

5. BOLMAN, W. - Theoretical and Empirical Bases of Community Mental Health. Amer. J. Psychiat. $124: 3,1967$ (supl.).

6. Caplan, G. - Principios de Psiquiatria Preventiva. Versåo castelhana. Ed. Paid6s, Buenos Aires, 1986. 
7. CAPLAN, G. - "Development of Community Psychiatry Concepts". In Comprehensive Textbook of Psychiatry, pág. 1516, editado por A. Freedman. The William \& Wilkins Co., New York, 1967.

8. Cerqueira, L. - Prioridades em Assistência Psiquiátrica. Coordenadoria de Saúde Mental de São Paulo, publicação no 7, São Paulo, 1973.

9. COElho, R. A. G. - Estrutura Social e Dinamica Psicologica. Editora Pioneira, Sð̊o Paulo, 1969.

10. DEVereuX, G. - A sociological theory of Schizophrenia. Psychoanal. Rev. 26: 315, 1939.

11 DREIKUS, R. - Early experiments in Social Psychiatry. Int. J. Soc. Psychiatry 7:141, 1961.

12. FIORINI, H. - Problematica actual de las psicoterapias breves. In Nuevas Perspectivas en Salud Mental, editado por W. Grimson. Versåo castelhana. Editorial Tiempo Comtemporaneo, Buenos Aires, 1973.

13. FOUCAULT, M. - Doença Mental e Paicológica. Versăo brasileira, Editora Tempo Brasileiro, Rio de Janeiro, 1968.

14. FregdmaN, - Community Psychiatry. British J. Psychiat. 114:481, 1968.

15. GOHFMAN, E. - Internados. Versão castelhana. Amorrortu Editores, Buenos Aires, 1972.

16. HALLECK, S. - Community Psychiatr: some troubling questions. In $L$. Roberts, edit. Community Psychiatry, Univ. of Wisconsin Press, Wisconsin, 196.

17. HEMPEL, C. G. - Fllosofia da Ciencia Natural. Versão brasileira. Zahar Editores, Rio de Janeiro, 1974.

18. HOLLINGSheAD, A. \& REDLICH, F. K. - Social Class and Mental Ilness. Science Exitions, New York, 1964.

19. JONES, M. - A Comunidade Terapêutica. Versăo brasileira. Ed. Vozes, Săo Paulo, 1972.

20. KUBIE, L. - Social forces and the neurotic process. In Explorations in Social Psychiatry, ed. por A. Lelghton. Tavistock Publications, Londres, 1957.

21. KUBIE, L. - Pitfalls of Community Psychiatry. Arch. Gen. Psychiat. 18:257, 1968.

22. LeightoN, A.; ClaUSEN, J. A. \& WILSON, R. N. - Explorations in Social Psychiatry, Tavistock Publications, Londres, 1957.

23. LEIGHTON, A. - Some propositions regarding the relacionship of sociocultural integration and disintegration to mental health. In Social Psychiatry, editado por Zubin. Grunne \& Stratton, New York, 1968.

24. LEVI, L. - Psychosocial stress and disease: a conceptual model. In Life Stress and Illness, editado por G. Gunderson. Gunderson. Charles C. Thomas, Springfield (Illinois), 1974.

25. LOEB, M. - Community Psychiatry: what it is and what is not. In Community Psychiatry, editado por L. Roberts. Univ: of Wisconsin Press, Wisconsin, 1966. 
26. MAIA, E: - Novas perspectivas da assistência psiquiátrica no Brasil. Rev. Med (Såo Paulo) 56:333, 1972.

27. MARTINS, C. - Programas de Psiquiatria Comunitária. Relatório oficial do V Congresso Latino-Americano de Psiquiatria, México, 1975.

28. MECHANIC, D. - Community Psychiatry: some sociological perspectives and implications. In Community Psychiatry, editado por L. Roberts. The Univ. of Wisconsin Press, Wisconsin, 1966.

29. MECHANIC, D. - Sociological Issues in Mental Health. In Progress in Community Mental Health, editado por L. Bellak. Grunne \& Stratton, New York, 1972.

30. MILLER, J. G. - Living systems: basic concepts (I). Behav. Sci. 10:193, 1965.

31. MILLER, J. G. - Living systems: basic concepts (II). Behav. Sci. 10:337, 1965.

32. MILLER, J. G. - Living systems: basic concepts (III). Behav. Sci. 10:380, 1965.

33. MILLS, Th. - Sociologia dos Pequenos Grupos. Versåo brasileira. Ed. Pioneira, 1970.

34. MORGAN, N. C. - Failures in Psychiatry: the chronic mental hospital. Am. J. Psychiat. 113:824, 1957.

35. NEGRETE, J. - Factores culturales en estudios epidemiologicos sobre alcoholismo. Acta Psichiat. Psicol. Amér. Lat. 20:112, 1974.

36. OMS, Ser. Inf. Tecn. 177 - Psiquiatria Social y Actitudes de la Coletividad, 1959.

37. OMS, Ser. Inf. Tecn. 185 - Epidemiologia de los Transtornos Mentales, 196c

38. OPAS, Publicaciones Cientificas 210 - Grupo de Trabajo sobre la Administracion de Servicios Psiquiatricos y de Salud Mental, 1970.

39. OSBONE, O. H. \& NAKAGAWA, H. - Distortions and Distinctions in Community Mental Health. Int. J. Soc. Psychiatry $19: 121,1974$.

40. PARSONS, T. - Sociocultural factors and personality. Psychiatry 21:321, 1958.

41. PIEDMONT, E. B. - Revolutions in psychiatry (or the emperor's new clothes). Int. J. Soc. Psychiatry 17:11, 1971.

42. RANSON, J. A. - Social Psychiatry: an overview. Amer. J. Psychiat. 130:841, 1973.

43. RENNIE, T. - Social Paychiatry: a definition. Int. J. Soc. Psychiatry 1:5, 1955.

44. ROBERTS, L. - Introduction in Community Psychiatry. The Univ. of Wiscosin, Wisconsin, 1966.

45. SABSHIN, M. - Theoretical Models in Community Psychiatry. In Community Psychiatry, ed. por L. Roberts. The Univ. of Wisconsin Press, Wisconsin, 1966.

46. SANDLER, P. C. - A Psiquiatria social e a epidemiologia dos distúrbios mentais. Rev. Med. (São Paulo) $65: 391,1971$. 
47. SANDLER, P. C. - Dinamica sócio-cultural na epidemiologia da esquizofrenia. Bol. Psiquiat. (São Paulo) 6:59, 1973.

48. SANDLER, P. C. - O papel da familia na doença mental. Revista do Hospital do Servidor Público de săo Paulo $-8: 170,1974$.

49. SANDLER, P. C. - Psiquiatria social: definição de termos e um esquema. Bol. Psiquiat. (São Paulo) 8:53, 1975.

50. SHWARTz, D. - Community Psychiatry in 72: an assesment. In Progress in Community Mental Health, editado por L. Bellak. Grunne \& Stratton, New York, 1972.

51. SHEPHERD, M. - Epidemiologia y Transtornos Mentales: uma revisión, Versåo castelhana. Caracas, 1969.

52. SIVADON, P. - Assistance Psychiatrique Communautaire. Relatório Oficial do VI Congresso Latino-Americano de Psiquiatria, 1970.

53. SROLE, L. - Mental Health in the Metropolis: the Midtown Manhattan Study. Mc-Graw-Hill, New York, 1962.

54. SROLE, L. - Social Psychiatry: a case of Babel syndrome. In Social Psychiatry. ed. por J. Zubin, Grunne \& Stratton, New York, 1968.

55. STEINHART, H. - The selling of community mental health. Psychiat. Quart. $47: 325,1973$.

56. WYNNE, L. C. - Family Research in the Pathogenesis of Schizophrenia. International Symposium on Psychosis, Montreal, 1969 (mimeogr.).

57. ZUBIN, J. \& HOCH. P. - Field Studies in Mental Disorders. Grunne \& Stratton, New York,. 1968.

58. ZUBIN, J. \& HOCH, P. - Social Psychiatry. Grunne \& Stratton, New York, 1968.

59. ZWERLING, I. - The Psychiatric Day Hospital. In American Handbook of Psychiatry, editado por Silvano Arietti. Basic Books New York, 1966.

Fraculdade de Saúde Pública da Universidade de săo Paulo - Saúde mental Av. Dr. Arnaldo 715 - 01855 sao Paulo, SP - Brasil. 